\title{
Zenker Diverticulum: Does Size Correlate with Preoperative Symptoms?
}

\author{
Jhon F. Martinez-Paredes ${ }^{1(0)}$ Razan Alfakir $\left.^{1(}\right)$ Jan L. Kasperbauer ${ }^{2(}$ Amy Rutt $^{10}$ \\ ${ }^{1}$ Department of Otolaryngology-Head and Neck Surgery, Mayo Clinic, \\ Jacksonville, Florida, United States \\ 2 Department of Otolaryngology-Head and Neck Surgery, Mayo Clinic, \\ Rochester, Minnesota, United States \\ Address for correspondence Jhon F. Martinez-Paredes, MD, \\ Department of Otolaryngology-Head and Neck Surgery, Mayo Clinic, \\ 4500 San Pablo Rd S, Jacksonville, FL 32224, United States \\ (e-mail: MartinezParedes.Jhon@mayo.edu).
}

Int Arch Otorhinolaryngol 2022;26(3):e334-e338.

\begin{abstract}
Introduction Zenker diverticulum (ZD) usually affects adults after the $7^{\text {th }}$ decade of life. Treatment for ZD is indicated for all symptomatic patients, but some patients prefer to defer surgical treatment until symptoms get worse and decrease their quality of life.

Objective To evaluate the association of the preoperative symptoms in ZD patients with the size of the ZD.

Methods A retrospective study design. Electronic medical records were used to identify patients diagnosed with ZD and treated over 11 years. Data collection included the chief complaints and symptoms, medical history, and findings on radiologic swallow evaluations of the patients. The diverticulum size was stratified into 3 groups: small $(<1 \mathrm{~cm})$, moderate $(1-3 \mathrm{~cm})$, and large $(>3 \mathrm{~cm})$.

Results A total of 165 patients were enrolled and stratified by diverticulum size ( 48 small, 67 medium, and 50 large). Dysphagia, cough, and regurgitation were the most prevalent symptoms. Dysphonia was more frequent among patients with a small pouch. Logistic regression analysis showed that dysphagia and choking were associat-

\section{Keywords}

- zenker diverticulum

- pharyngeal pouch

- esophageal diverticulum

- dysphagia

- dysphonia ed with large and medium diverticulum size $(p<0.05)$. Additionally, dysphonia was significantly associated with the presence of a small-sized ZD $(p<0.04)$.

Conclusion Upper gastrointestinal symptoms such as dysphagia and choking may be associated with a ZD $>1 \mathrm{~cm}$ and should always be evaluated. Additionally, the presence of dysphonia was found to be correlated with a ZD $<1 \mathrm{~cm}$, suggesting that a prompt and appropriate fluoroscopic evaluation must be considered in those patients in whom no other clear cause of dysphonia is evident.
\end{abstract}

\section{Introduction}

Zenker diverticulum (ZD) or pharyngeal pouch is a rare disorder affecting 2 in every 100,000 people. The incidence is male predominant (ratio 1:5) and usually occurs in middle-aged adults and older adults in the $7^{\text {th }}$ or $8^{\text {th }}$ decades of life. The etiology of ZD remains controversial; however, the presence of a structural or functional abnormality of the cricopharyngeus muscle plays an important role. ${ }^{1-3}$ Patients presenting to the otolaryngology clinic with ZD often have received

November 17, 2020 accepted after revision

February 14, 2021

published online

October 26, 2021
DOI https://doi.org/ 10.1055/s-0041-1730457. ISSN 1809-9777.

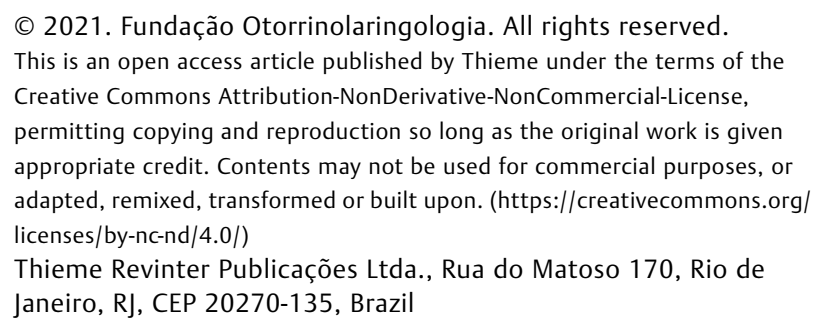


multiple complaints. The co-occurrence of pathologies such as gastroesophageal reflux disease (GERD) or hiatal hernia makes the clinical evaluation of these patients a clinical challenge to decipher which swallowing complaints are related to the $\mathrm{ZD} .^{4}$

Common clinical presentations include dysphagia, regurgitation, choking, chronic cough, aspiration pneumonitis, globus, weight loss and, less commonly, dysphonia. ${ }^{5}$ Clear clinical history and physical examination, associated with positive findings on radiologic swallow evaluation - modified barium swallow study (MBSS) and barium esophagram - are crucial for the diagnosis and size classification of ZD. ${ }^{6}$ According to Van Overbeek the ZD size can be classified using a radiological scale into a small pouch when it is shorter than one vertebra, and into a large pouch when it is longer than three vertebrae. ${ }^{7}$ This classification provides a better understanding of the correlation of the symptoms of the patient with diverticulum size to improve clinical insight in diagnosis, treatment planning, and preoperative counseling.

Treatment for ZD is indicated for all symptomatic patients, but some patients prefer to defer surgical treatment until symptoms get more persistent and decrease their quality of life. ${ }^{8}$ Some studies have evaluated the relation of some clinical symptoms, such as globus and regurgitation, with diverticulum size in patients with ZD who underwent surgical procedures. However, studies that assessed the preoperative clinical presentations with diverticulum size in $\mathrm{ZD}$ patients are currently lacking. The present study aims to evaluate the association of the preoperative symptoms in ZD patients with the diverticulum size.

\section{Methods}

A retrospective study design approved by the Institutional Review Board (IRB 20-003440) was conducted. Electronic medical records (EMR) were used to identify patients diagnosed with ZD (ICD-10: K22.5 diverticulum of the esophagus, acquired) from January 2009 through April 2020. A total of 165 patients were identified and enrolled after the inclusion and exclusion criteria were applied. A chart review of clinical data, including the first visit to the otolaryngology clinic, previous medical history, and findings on the radiologic swallow evaluation with MBSS or a barium esophagram, was completed. The initial medical evaluation included the assessment of the presenting complaint and symptoms, including dysphagia, odynophagia, dysphonia, cough, choking, regurgitation, aspiration, globus, and weight loss. The intraoperative reports were also examined to evaluate the ZD size and the method of treatment. The ZD size was stratified into 3 groups: small $(<1 \mathrm{~cm})$, moderate $(1-3 \mathrm{~cm})$, and large $(>3 \mathrm{~cm})$. Surgical treatment included open diverticulectomy with or without cricopharyngeal myotomy, endoscopic $\mathrm{CO} 2$ laser or staple diverticulectomy, and cricopharyngeal myotomy. All the data were collected from the medical record and stored in a RedCap database.

Statistical analyses were performed using IBM SPSS Statistics for Windows, version 25 (IBM Corp., Armonk, NY, USA). Standard descriptive statistics were obtained and presented as percentages, mean \pm standard deviations (SDs). A parametric statistical analysis (student $t$-test) was used to compare demographics and comorbidities between the groups of patients. Additionally, a Pearson correlation and a logistic regression analysis were also performed to evaluate the association of presenting symptoms (independent variables) and the $\mathrm{ZD}$ size (dependent variable). A $p$-value $<0.05$ was considered statistically significant.

\section{Results}

The mean age of the patients among the overall cohort was 73 years old (range 39-96 years old) with a 1.2:1 male/female ratio. Pre-existing GERD diagnosis was present in $65.5 \%$ of the patients, and $43.5 \%$ of the patients reported a history of tobacco consumption. Thirty-eight patients had a diagnosis of hiatal hernia (23\%). There was no difference in age, gender distribution, body mass index (BMI), frequency of hiatal hernia, and comorbidities between groups $(p>0.05)$. The demographic summary of ZD patients is shown in - Table 1.

Small ZD size $(<1 \mathrm{~cm})$ was found in 48 patients, medium size $(1-3 \mathrm{~cm})$ in 67 patients, and large size $(>3 \mathrm{~cm})$ in 50 patients. The prevalence of symptoms per ZD size is shown in -Table 2. Dysphagia was the most prevalent symptom among all patients (89.1\%), followed by cough (65.5\%) and regurgitation (58.8\%). Dysphonia was a more frequent symptom among patients with a small ZD (22\%) than in those with a medium and large diverticulum ( 9 and $4 \%$, respectively).

Table 1 Demographic Summary and Clinical characteristics, overall cohort.

\begin{tabular}{|l|l|}
\hline Characteristics & $n=165$ \\
\hline Gender, No. (\%), male & $92(55.8)$ \\
\hline Mean age, y (range, SD) & $73(39-96,11)$ \\
\hline BMI kg/m², mean (SD) & $26.9(5.6)$ \\
\hline Tobacco consumption, No. (\%) & $71(43.5)$ \\
\hline EtOH consumption, No. (\%) & $56(34.3)$ \\
\hline Radiologic evaluation & \\
\hline MBSS, No. (\%) & $108(67.5)$ \\
\hline Esophagram, No. (\%) & $146(90.6)$ \\
\hline Comorbidities & \\
\hline Hypertension, No. (\%) & $86(52.1)$ \\
\hline Diabetes Mellitus, No. (\%) & $19(11.5)$ \\
\hline Dyslipidemia, No. (\%) & $75(45.5)$ \\
\hline GERD, No. (\%) & $108(65.5)$ \\
\hline OSA, No. (\%) & $18(11)$ \\
\hline Hiatal Hernia, No. (\%) & $38(23)$ \\
\hline
\end{tabular}

Abbreviations: BMI, Body Mass Index; EtOH, Ethanol; GERD, Gastroesophageal reflux disease; MBSS, Modified barium swallow study; OSA, Obstructive sleep apnea; SD, standard deviation. 
Table 2 Prevalence of symptoms according to Zenker Diverticulum size

\begin{tabular}{|c|c|c|c|c|c|c|c|c|}
\hline & \multicolumn{2}{|l|}{ All } & \multicolumn{2}{|c|}{ Small } & \multicolumn{2}{|c|}{ Medium } & \multicolumn{2}{|c|}{ Large } \\
\hline & $n$ & $\%$ & $n$ & $\%$ & $n$ & $\%$ & $n$ & $\%$ \\
\hline Dysphagia & 147 & $89.1 \%$ & 37 & $77.1 \%$ & 64 & $95.5 \%$ & 46 & $92 \%$ \\
\hline Cough & 108 & $65.5 \%$ & 30 & $62.5 \%$ & 44 & $65.7 \%$ & 34 & $68 \%$ \\
\hline Regurgitation & 97 & $58.8 \%$ & 23 & $47.9 \%$ & 44 & $65.7 \%$ & 30 & $60 \%$ \\
\hline Globus & 80 & $48.5 \%$ & 20 & $41.7 \%$ & 31 & $46.3 \%$ & 29 & $58 \%$ \\
\hline Choking & 61 & $37 \%$ & 8 & $16.7 \%$ & 27 & $40.3 \%$ & 26 & $52 \%$ \\
\hline Aspiration & 31 & $18.8 \%$ & 7 & $14.6 \%$ & 16 & $23.9 \%$ & 8 & $16 \%$ \\
\hline Dysmotility & 23 & $13.9 \%$ & 6 & $12.5 \%$ & 8 & $11.9 \%$ & 9 & $18 \%$ \\
\hline Weight loss & 20 & $12.1 \%$ & 2 & $4.2 \%$ & 9 & $13.4 \%$ & 9 & $18 \%$ \\
\hline Dysphonia & 19 & $11.5 \%$ & 11 & $22.9 \%$ & 6 & $9 \%$ & 2 & $4 \%$ \\
\hline Odynophagia & 11 & $6.7 \%$ & 3 & $6.3 \%$ & 4 & $6 \%$ & 4 & $8 \%$ \\
\hline
\end{tabular}

Table 3 Pearson correlation between presenting symptoms and Zenker Diverticulum size

\begin{tabular}{|l|l|l|}
\hline & $\boldsymbol{r}$ & $p$-value \\
\hline Dysphagia & 0.18 & 0.019 \\
\hline Choking & 0.28 & 0.001 \\
\hline Weight loss & 0.16 & 0.037 \\
\hline Dysphonia & -0.22 & 0.003 \\
\hline
\end{tabular}

\section{Correlation Coefficients}

Pearson correlation between presenting symptoms and ZD size was performed and is shown in - Table 3. A small but statistically significant positive correlation was found between ZD size and choking $(r=0.28 ; p=0.001)$, dysphagia $(r=0.18 ; p=0.019)$, and weight loss $(r=0.16 ; p=0.037)$. Also, a statistically significant negative correlation between ZD size and dysphonia ( $r=-0.22 ; p=0.003$ ) was noted.

\section{Logistic Regression}

Our logistic regression model showed a statistically significant relationship between the presence of dysphagia and choking with the presence of a medium-sized and largesized ZD when compared with small-sized diverticula $(p<0.05)$. The odds ratio (OR) of dysphagia to be associated with a medium diverticula was 9.7 (95\% confidence interval (CI): $2.0-46.1 ; p=0.004)$ and the OR associated with a largesized diverticula was 6.0 (95\%CI: $1.3-27.0 ; p=0.018$ ). The OR of choking associated with a medium diverticula was 4.8 (95\%CI: $1.5-15.2 ; p=0.008)$, and with large diverticula it was 5.6 (95\%CI: $1.8-17.5 ; p=0.003)$. Although dysphonia was the least frequent symptom among the three groups of patients, its presence was significantly associated with the presence of a small-sized ZD when compared with a bigger ZD $(p<0.04)$. The full details of our logistic regression analysis are presented in - Table 4.

\section{Discussion}

The present study evaluated the presence of preoperative symptoms among a large cohort of patients with a diagnosis of ZD and its association with the documented diverticulum size. Our population showed an elderly male predominance, agreeing with the data found in several cohort studies. ${ }^{9-13}$ The coprevalence of GERD and hiatal hernia was also evident in our sample. These two pathologies have been commonly reported as co-occurring conditions in individuals with $\mathrm{ZD}$, making their symptoms easily confused with the presence or recurrence of a pharyngeal pouch. ${ }^{10,14,15}$ This finding confirms the importance of early treatment, as the presence of ZD could lead to a nutritional compromise and to a potential escalating comorbidity over time. ${ }^{16}$ Our results showed that dysphagia, cough, regurgitation, and globus are the most prevalent symptom among all groups.

Interestingly, a global incidence of dysphonia of $11.5 \%$ was also found among our patients. This is consistent with other studies (Palmer et al., 2007; Bergeron et al., 2013; Greene et al., 2015). 9,10,12 For example, Greene et al. reported dysphagia caused by outflow resistance in the esophagus as the primary preoperative symptom in their cohort of 77 patients, followed by regurgitation and cough. ${ }^{12}$ Palmer et al. surveyed symptoms before and after the endoscopic repair of the $\mathrm{ZD}$ among a group of 72 patients, finding that food avoidance, regurgitation, choking, and cough were the most prevalent clinical manifestations. ${ }^{10} \mathrm{~A}$ difference in the study design (i.e., both pre- and postsurgical repair were included relative to our study) could explain the difference in the results.

Our data also indicates that dysphagia and choking are significantly associated with the presence of a medium-sized or large-sized pharyngeal pouch. Bergeron et al. evaluated the characteristics of dysphagia and its correlation with the ZD size within a cohort of 46 patients. Their findings were similar to our data, suggesting the relationship between dysphagia and regurgitation with a medium or large $\mathrm{ZD}$ size, and highlighting the importance of a radiologic swallow evaluation if patients with a history of ZD removal persist 
Table 4 Logistic regression model between clinical symptoms and Zenker Diverticulum size

\begin{tabular}{|c|c|c|c|c|}
\hline & $\beta$-value & Standard Error & OR $(95 \% \mathrm{Cl})$ & $p$-value \\
\hline \multicolumn{5}{|l|}{ Dysphagia } \\
\hline Small $(>1 \mathrm{~cm})$ & Reference & & & \\
\hline Medium $(1-3 \mathrm{~cm})$ & 2.27 & 0.79 & $9.75(2.06-46.16)$ & $0.004^{*}$ \\
\hline Large $(>3 \mathrm{~cm})$ & 1.80 & 0.76 & $6.06(1.36-27.04)$ & $0.018^{*}$ \\
\hline \multicolumn{5}{|l|}{ Choking } \\
\hline Small $(>1 \mathrm{~cm})$ & Reference & & & \\
\hline Medium $(1-3 \mathrm{~cm})$ & 1.57 & 0.58 & $4.81(1.51-15.28)$ & $0.008^{*}$ \\
\hline Large $(>3 \mathrm{~cm})$ & 1.72 & 0.58 & $5.63(1.8-17.56)$ & $0.003^{*}$ \\
\hline \multicolumn{5}{|l|}{ Dysphonia } \\
\hline Small $(>1 \mathrm{~cm})$ & Reference & & & \\
\hline Medium $(1-3 \mathrm{~cm})$ & -1.34 & 0.64 & $0.26(0.74-0.91)$ & $0.036^{*}$ \\
\hline Large $(>3 \mathrm{~cm})$ & -1.99 & 0.84 & $0.13(0.02-0.75)$ & $0.023^{*}$ \\
\hline \multicolumn{5}{|l|}{ Regurgitation } \\
\hline Small $(>1 \mathrm{~cm})$ & Reference & & & \\
\hline Medium $(1-3 \mathrm{~cm})$ & 0.27 & 0.49 & $1.31(0.5-3.46)$ & 0.576 \\
\hline Large $(>3 \mathrm{~cm})$ & 0.04 & 0.52 & $1.04(0.37-2.91)$ & 0.939 \\
\hline \multicolumn{5}{|l|}{ Globus } \\
\hline Small $(>1 \mathrm{~cm})$ & Reference & & & \\
\hline Medium $(1-3 \mathrm{~cm})$ & -0.41 & 0.49 & $0.65(0.24-1.73)$ & 0.398 \\
\hline Large $(>3 \mathrm{~cm})$ & 0.11 & 0.53 & $1.11(0.39-3.17)$ & 0.833 \\
\hline
\end{tabular}

with dysphagia. ${ }^{9}$ However, our study benefited from having a larger sample size and not previously analyzed symptoms such as dysmotility, dysphonia, weight loss, and other medical history variables.

Only one study, by Schoeff et al., has identified dysphonia as a disease feature among patients with ZD. Although they found dysphonia as an unrecognized preoperative complaint, a significant improvement in the voice handicap index-10 (VHI-10) before and after surgery was noted. ${ }^{17}$ Our results correlate with this study, as the incidence of dysphonia within the groups of patients with a mediumsized and large-sized diverticulum was also low. We hypothesize that the misperception and underestimation of voice handicap among individuals with ZD can explain this finding. We also hypothesize that the presence of dysphonia among patients with a small pouch could have caused an early endoscopic evaluation and diagnosis during the study of more prevalent causes of dysphonia, including GERD, more than the ZD per se. It is noteworthy that radiologic swallow evaluation with MBSS or a barium esophagram has been widely recommended for patients with suspected structural abnormalities, including ZD. ${ }^{6,18}$ Our results contribute to the literature and can be the starting point to consider a fluoroscopic swallowing evaluation among those patients in whom no other apparent cause of dysphonia is documented.

\section{Limitations}

As a retrospective study, there are several intrinsic limitations to consider. For example, cointerventions and confounders could not be controlled due to the nature of our study. In some medical records, limited data were available, including notes regarding the improvement of symptoms after the surgical repair of the ZD and voice-related questionnaires. We consider that further prospective studies with larger sample sizes and a complete preoperative and postoperative evaluation of symptoms will increase the statistical power. Additionally, to confirm our findings, studies involving the preoperative and postoperative assessment of patient-reported outcome measures, including the VHI-10, Glottal Function Index (GFI), Reflux Symptoms Index (RSI), and the Voice-Related Quality of Life score (VRQOL), are needed.

\section{Conclusions}

Our study suggests that upper gastrointestinal symptoms such as dysphagia and choking may be associated with a $\mathrm{ZD}>1 \mathrm{~cm}$ and should always be evaluated. Additionally, the presence of dysphonia was found to correlate with a $\mathrm{ZD}<1$ $\mathrm{cm}$, suggesting that a prompt and appropriate fluoroscopic evaluation must be considered in those patients in whom no other clear cause of dysphonia is evident. 
338 Zenker Diverticulum: Does Size Correlate With Preoperative Symptoms? Martinez-Paredes et al.

\section{Funding \\ None.}

\section{Conflict of Interests}

The authors have no conflict of interests to declare.

\section{References}

1 Cook IJ, Blumbergs P, Cash K, Jamieson GG, Shearman DJ. Structural abnormalities of the cricopharyngeus muscle in patients with pharyngeal (Zenker's) diverticulum. J Gastroenterol Hepatol 1992;7(06):556-562

2 Lerut T, Coosemans W, Decaluwé H, et al.Zenker's diverticulum. Multimedia manual of cardiothoracic surgery: MMCTS. 2009; 2009(224):mmcts.2007.002881.

3 Visser LJ, Hardillo JA, Monserez DA, Wieringa MH, Batenburg de Jong RJ. Zenker's diverticulum: Rotterdam experience. Eur Arch Otorhinolaryngol 2016;273(09):2755-2763

4 Morales-Divo C, Jecker P, Lippert B, Mann WJ. [Extraesophageal reflux in patients suffering from Zenker's diverticulum]. HNO 2007;55(07):546-550

5 Siddiq MA, Sood S, Strachan D. Pharyngeal pouch (Zenker's diverticulum). Postgrad Med J 2001;77(910):506-511

6 Nehring P, Krasnodębski IW. Zenker's diverticulum: aetiopathogenesis, symptoms and diagnosis. Comparison of operative methods. Prz Gastroenterol 2013;8(05):284-289

7 van Overbeek JJ. Meditation on the pathogenesis of hypopharyngeal (Zenker's) diverticulum and a report of endoscopic treatment in 545 patients. Ann Otol Rhinol Laryngol 1994;103(03): $178-185$
8 Ferreira LE, Simmons DT, Baron TH. Zenker's diverticula: pathophysiology, clinical presentation, and flexible endoscopic management. Dis Esophagus 2008;21(01):1-8

9 Bergeron JL, Long JL, Chhetri DK. Dysphagia characteristics in Zenker's diverticulum. Otolaryngol Head Neck Surg 2013;148 (02):223-228

10 Palmer AD, Herrington HC, Rad IC, Cohen JI. Dysphagia after endoscopic repair of Zenker's diverticulum. Laryngoscope 2007;117(04):617-622

11 Shah RN, Slaughter KA, Fedore LW, Huang BY, Deal AM, Buckmire RA. Does residual wall size or technique matter in the treatment of Zenker's diverticulum? Laryngoscope 2016;126(11): 2475-2479

12 Greene CL, McFadden PM, Oh DS, Chang EJ, Hagen JA. Long-Term Outcome of the Treatment of Zenker's Diverticulum. Ann Thorac Surg 2015;100(03):975-978

13 Périé S, Dernis HP, Monceaux G, Angelard B, St Guily JL. The "sign of the rising tide" during swallowing fiberoscopy: a specific manifestation of Zenker's diverticulum. Ann Otol Rhinol Laryngol 1999;108(03):296-299

14 Cote DN, Miller RH. The association of gastroesophageal reflux and otolaryngologic disorders. Compr Ther 1995;21(02):80-84

15 Sen P, Kumar G, Bhattacharyya AK. Pharyngeal pouch: associations and complications. Eur Arch Otorhinolaryngol 2006;263 (05):463-468

16 Beard K, Swanström LL. Zenker's diverticulum: flexible versus rigid repair. J Thorac Dis 2017;9(Suppl 2):S154-S162

17 Schoeff S, Freeman M, Daniero J. Voice Outcomes in Surgical Repair of Zenker's Diverticulum. Dysphagia 2017;32(05):678-682

18 Allen BC, Baker ME, Falk GW. Role of barium esophagography in evaluating dysphagia. Cleve Clin J Med 2009;76(02):105-111 